\title{
Consumo de lácteos en población universitaria de la ciudad de Medellín
}

\author{
Dairy consumption of university \\ students at Medellin city
}

\begin{abstract}
Intake of dairy products in a population of 420 college students from 4 universities of Medellin city, Colombia, was evaluated to determine consumption preferences and knowledge through a personal interview. Analysis of the data using the multivariate technique MANOVA with canonical orthogonal contrast was performed using the statistical package SAS version 9.0. $80.7 \%$ of the students consumed milk, with a highly significant difference in the frequency of consumption among socioeconomic levels, being the highest consumption at the highest level. A significant difference in milk consumption according to gender was found, with a higher consumption by men. Milk ranks fifth as favorite beverage $(6.1 \%)$, behind juice, water, soft drink and aromatic drinks. Cost (28.3\%) and taste (20.9\%) were the main reasons for non-consumption of dairy milk.

Key words: dairy consumption, university students, feeding habits.
\end{abstract}

Fernando Restrepo B. Holmes Rodríguez $E$. Joaquín Angulo A.

Grupo GRICA, Facultad de Ciencias Agrarias, Universidad de Antioquia, Medellín, Colombia.

$$
\begin{array}{r}
\text { Dirigir la correspondencia a: } \\
\text { Profesor } \\
\text { Fernando Restrepo B. } \\
\text { Grupo GRICA } \\
\text { Facultad de Ciencias Agrarias } \\
\text { Universidad de Antioquia } \\
\text { Calle } 70 \text { No. 52-21, } \\
\text { Medellín, Colombia } \\
\text { E-mail: frbstatistical@yahoo.com }
\end{array}
$$

Este trabajo fue recibido el 31 de Marzo de 2014 y aceptado para ser publicado el 20 de Noviembre de 2014.

\section{INTRODUCCIÓN}

La leche y sus derivados son alimentos de alto valor nutricional, fuentes de proteínas, vitaminas y minerales (1), especialmente de calcio, por lo cual la ingesta de lácteos está relacionada con el contenido de este mineral en el tejido óseo (2). Las ventajas del consumo de lácteos para la salud, han sido ampliamente documentadas en la literatura científica, entre las cuales se cuentan la protección contra el riesgo de sufrir de obesidad (3); reducción del riesgo de baja masa ósea, infarto, síndrome metabólico y algunos tipos de cáncer $(4,5)$; reducción de la presión sanguínea en jóvenes (6); reducción de factores de riesgo de enfermedades crónicas (7) y reducción en la prevalencia de caries dental (8).

En Colombia, el consumo estimado de leche alcanza los 156 L/persona/año (9), cantidad inferior a la recomendación de la Organización Mundial de la Salud-OMS (10) de 170 L/persona/año y del consumo en otros países de la región como Uruguay que alcanza los $230 \mathrm{~L} /$ persona/año, seguido de Argentina con 220 L/persona/año. De acuerdo con los hallazgos de la Encuesta Nacional de la Situación Alimentaria y Nutricional (ENSIN) (11) el consumo de leche líquida en población colombiana de 19 a 50 años es de 226,8 cc/niño/día, ubicándose por debajo del consumo de gaseosas y refrescos que alcanza los 405,8 cc/niño/día. Frente a esta situación, estudios han encontrado que consumos bajos de productos lácteos están asociados con deficiencias de calcio y magnesio en ambos sexos (12).
Adicionalmente, en Colombia se viene incrementando el sobrepeso y la obesidad de la población, que pasó de $46 \%$ en 2005, a 52\% en 2010 (13), por lo cual es necesario implementar estrategias que permitan mejorar la ingesta de productos lácteos. Por tal razón, el objetivo de este estudio fue determinar los hábitos de consumo de productos lácteos en población universitaria que permitan identificar aspectos relevantes para incentivar su consumo.

\section{SUJETOS Y MÉTODOS}

Para llevar a cabo el proceso investigativo se diseñó una encuesta de tipo estructurado de acuerdo con los objetivos planteados, teniendo en cuenta variables de tipo cualitativo asociado a patrones probabilísticos, multinomial y binomial: tipo de lácteos consumidos, frecuencia de consumo, tipo de quesos consumidos, conocimientos sobre los nutrientes que aporta la leche y razones para no consumir lácteos. La encuesta se sometió a juicio de expertos con el objetivo de verificar la pertinencia de las preguntas y de la metodología propuesta.

La técnica de muestreo empleada fue muestreo aleatorio de proporciones en forma doblemente estratificada; por género y tipo de universidad. Se utilizó un nivel de confiabilidad del $95 \%$ y un error máximo permisible del $4.8 \%$, donde los parámetros estimados $\mathrm{P}$ y $\mathrm{Q}$ adoptaron el valor del $50 \%$ debido a que no se poseen estudios anteriores sobre el consumo de lácteos en la población objeto de estudio, cabe 
anotar que dichos valores permiten maximizar el tamaño de la muestra. La edad de los estudiantes osciló entre los 18 a 24 años. El tamaño definitivo de la muestra fue 420 estudiantes pertenecientes a universidades públicas y privadas. La muestra se eligió en forma aleatoria condicionada de acuerdo con el criterio de estratificación antes citado, utilizando una distribución uniforme.

La encuesta fue aplicada con la participación voluntaria de los estudiantes. La base de datos se elaboró en una hoja electrónica de acuerdo con el formato delineado en la encuesta y se realizó un estricto control de calidad en tres etapas del proceso investigativo, con el fin de garantizar los resultados y conclusiones generadas a la luz de la información.

El análisis estadístico de los datos se realizó con el paquete estadístico SAS versión 9.0. Se empleó análisis multivariado de la varianza MANOVA con contraste canónico ortogonal, estableciendo la dimensionalidad de la comparación multidimensional, por medio del criterio de máxima verosimilitud observando el mayor valor propio significativo`. El estudio se efectuó con variables respuestas de tipo cualitativo expresadas en porcentaje. El análisis se complementó por medio de distribuciones de frecuencia de tipo univariado y bivariado mediante tablas de contingencia.

\section{RESULTADOS}

De los estudiantes universitarios indagados, 80,2\% había tomado leche entera, $67,2 \%$ leche en polvo, $54,4 \%$ leche saborizada, $34,1 \%$ leche deslactosada y $26,3 \%$ leche cruda. Los tipos de leches que menos consumían eran: la que contiene fitoesteroles $(1,9 \%)$ y la que contiene omega $3(2,2 \%)$. La leche entera es la de mayor frecuencia de consumo diario $(60,2 \%)$, seguida de la deslactosada con 20,8\% (tabla 1).

La leche en polvo, saborizada, semidescremada y descremada tienen mayor consumo en el estrato alto; la leche entera y deslactosada en el estrato medio, mientras el mayor porcentaje de consumo de leche cruda se da en el estrato económico de menor poder adquisitivo, en este estrato las leches descremadas son las de menor consumo. La mayor frecuencia de consumo diario de leche entera, deslactosada y cruda se da en estudiantes universitarios de estrato alto. La leche en polvo tiene mayor frecuencia de consumo diario en el estrato bajo. En las universidades privadas se consume una mayor variedad de leche que en las públicas (tabla 1).

Se detectó una diferencia altamente significativa entre estratos y entre tipos de universidad pública y privada referente al tipo de leche consumida $(p<0,001)$; No se encontró diferencia $(p=0,1625)$ en el tipo de leche consumida entre géneros (tabla 2). Se detectó una diferencia altamente significativa entre estratos y entre tipos de universidad pública y privada referente a frecuencia de consumo de leche consumida $(p<0,001)$; los estudiantes del estrato alto presentaban una mayor frecuencia de consumo que los del bajo. No se encontró diferencia $(p=0,1014)$ en la frecuencia de consumo entre géneros.

En cuanto al horario, el mayor consumo de leche se da en la mañana $(56,7 \%)$, seguido de la tarde $(29,1 \%)$ y en menor proporción en la noche $(14,2 \%)$. Frente al tipo de leche consumida y teniendo en cuenta que un estudiante puede consumir más de un tipo de leche, 95,8\% de los indagados consume leche de vaca, 35,9\% leche de soya, 31\% leche de cabra y 10,7\% leche de búfala. En términos del lugar donde se más se recalca la importancia que poseen dichos lácteos, el hogar fue el principal $(60,2 \%)$, seguido de la escuela (21\%), la universidad $(12,4 \%)$ y en menor proporción los medios de comunicación $(6,4 \%)$. No se detectó diferencia por género $(p=0,2564)$, ni entre estratos $(p=0,1452)$ relacionado con la hora de consumo de los lácteos.

Al preguntar sobre la bebida preferida, se estableció que los estudiantes prefieren los jugos $(16,2 \%)$, seguidos del agua $(14,4 \%)$, gaseosa $(9,5 \%)$, aromáticas $(7 \%)$, leche $(6,1 \%)$, café $(5,3 \%)$ mientras que las bebidas energizantes constituyen la bebida de menor preferencia (5\%).

En cuanto a las preferencias del consumo de alimentos o bebidas como acompañantes de la leche, se encontró que $82,5 \%$ de los estudiantes evaluados prefieren el café como acompañante de la leche, 79,1\% las tortas, 76,3\% jugos preparados en leche, $66,8 \%$ los postres, $66 \%$ los chocolates y $61,3 \%$ los dulces.

En general, los hombres consumen derivados lácteos en mayor porcentaje que las mujeres y es en el estrato alto donde

\section{TABLA 1}

Consumo y frecuencia de consumo de distintos tipos de leche

\begin{tabular}{|c|c|c|c|c|c|c|c|c|c|c|}
\hline \multirow[b]{2}{*}{ Tipo leche } & \multirow{2}{*}{$\begin{array}{l}\text { Consumo } \\
\qquad \%\end{array}$} & \multicolumn{3}{|c|}{$\begin{array}{l}\text { Frecuencia de } \\
\text { consumo }\end{array}$} & \multicolumn{3}{|c|}{$\begin{array}{l}\text { Consumo por nivel } \\
\text { socioeconómico }\end{array}$} & \multicolumn{3}{|c|}{$\begin{array}{l}\text { Frecuencia por nivel } \\
\text { socioeconómico }\end{array}$} \\
\hline & & Diario & Semanal & Rara vez & Bajo & Medio & Alto & Bajo & Medio & Alto \\
\hline Entera & 80,2 & $60,2 \mathrm{a}$ & $25,1 \mathrm{~b}$ & $14,7 c$ & $76,8 \mathrm{a}$ & $82,1 \mathrm{a}$ & $76,7 a$ & $65,4 a$ & $56,8 b$ & $69,0 a$ \\
\hline Descremada & 26,3 & $19,2 \mathrm{c}$ & $35,5 b$ & $45,3 \mathrm{a}$ & $20,2 b$ & $25,9 b$ & $35,7 a$ & $18,7 a$ & $19,7 a$ & $18,1 \mathrm{a}$ \\
\hline Deslactosada & 34,1 & $20,8 c$ & $32,0 \mathrm{~b}$ & $47,2 \mathrm{a}$ & $33,3 a$ & $34,8 a$ & $32,1 \mathrm{a}$ & $14,6 b$ & $21,6 a$ & $22,2 \mathrm{a}$ \\
\hline Polvo & 67,2 & $12,7 \mathrm{c}$ & $40,4 a$ & $46,9 \mathrm{a}$ & $62,3 b$ & $65,9 b$ & $79,0 a$ & $14,2 \mathrm{a}$ & $12,5 a$ & $11,9 a$ \\
\hline Semidescremada & 20,5 & $13,3 \mathrm{c}$ & $32,0 \mathrm{~b}$ & $54,7 \mathrm{a}$ & $11,5 c$ & $20,8 b$ & $30,3 a$ & $10,0 a$ & $14,5 a$ & $11,7 a$ \\
\hline Con omega 3 & 2,2 & $16,6 \mathrm{c}$ & $33,0 \mathrm{~b}$ & $50,4 a$ & & & & & & \\
\hline Fitoesteroles & 1,9 & $6,6 c$ & $40,0 \mathrm{~b}$ & $53,4 a$ & & & & & & \\
\hline Saborizada & 54,4 & $3,6 \mathrm{c}$ & $31,9 b$ & 64,5 a & $44,9 c$ & $54,8 b$ & $64,2 a$ & $10,0 a$ & $3,1 b$ & $0,0 b$ \\
\hline Cruda & 26,3 & $9,5 b$ & $9,5 \mathrm{~b}$ & $81,0 \mathrm{a}$ & $37,6 a$ & $25,9 b$ & $14,2 c$ & $16,0 b$ & $3,3 c$ & $30,0 a$ \\
\hline
\end{tabular}

Letras distintas indican diferencia significativa en consumo y frecuencia de consumo por tipo de leche entre niveles socioeconómicos ( $p<0,05$ ).

Se empleó el modelo lineal general (GLM). 
se observa un mayor consumo de estos productos (tabla 3).

Se detectó una diferencia altamente significativa entre géneros, estratos y entre tipos de universidad pública y privada referente al consumo de derivados lácteos $(p<0,001)$. Los estudiantes del estrato alto consumen más derivados lácteos que los del estrato bajo (tabla 2). El estrato 3 en general es el de mayor consumo de los distintos tipos de queso, de igual manera se puede apreciar que el género masculino tiene más participación en el consumo de los distintos tipos de queso (tabla 4).

Se detectó una diferencia altamente significativa entre estratos y entre tipos de universidad pública y privada referente al consumo de quesos $(p<0,001)$. La diferencia radicó entre el estrato alto y el bajo. No se detectó diferencia $(p=0,4069)$

TABLA 2

Análisis multivariado de la varianza comparativo entre géneros, estratos y tipos de universidad.

\begin{tabular}{|c|c|c|c|c|c|c|}
\hline \multirow{3}{*}{$\begin{array}{l}\text { Prueba } \\
\text { Estadística }\end{array}$} & \multicolumn{3}{|c|}{ Consumo de leche } & \multicolumn{3}{|c|}{ Frecuencia de consumo de leche } \\
\hline & \multicolumn{3}{|c|}{ Valor de $\mathrm{p}$} & \multicolumn{3}{|c|}{ Valor de $\mathrm{p}$} \\
\hline & Género & Estrato & Universidad & Género & Estrato & Universidad \\
\hline Wilks' Lambda & 0,1625 & 0,0038 & 0,0004 & 0,1014 & 0,0025 & 0,0007 \\
\hline Pillai's Trace & 0,1625 & 0,0042 & 0,0004 & 0,1014 & 0,0047 & 0,0007 \\
\hline Hotelling-Lawley T & 0,1625 & 0,0037 & 0,0004 & 0,1014 & 0,0025 & 0,0007 \\
\hline Roy's Greatest R & 0,1625 & 0,0004 & 0,0004 & 0,1014 & 0,0005 & 0,0007 \\
\hline & \multicolumn{3}{|c|}{ Consumo de derivados lácteos } & \multicolumn{3}{|c|}{ Frecuencia de quesos } \\
\hline Prueba & \multicolumn{3}{|c|}{ Valor de $p$} & \multicolumn{3}{|c|}{ Valor de $p$} \\
\hline Estadística & Género & Estrato & Universidad & Género & Estrato & Universidad \\
\hline Wilks' Lambda & 0,0025 & 0,0091 & 0,0001 & 0,4069 & 0,0002 & 0,0001 \\
\hline Pillai's Trace & 0,0025 & 0,0091 & 0,0001 & 0,4069 & 0,0002 & 0,0001 \\
\hline Hotelling-Lawley T & 0,0025 & 0,0091 & 0,0001 & 0,4069 & 0,0002 & 0,0001 \\
\hline Roy's Greatest R & 0,0025 & 0,0091 & 0,0001 & 0,4069 & 0,0002 & 0,0001 \\
\hline & \multicolumn{3}{|c|}{ Frecuencia consumo quesos } & \multicolumn{3}{|c|}{ Conocimientos valor nutricional } \\
\hline Prueba & \multicolumn{3}{|c|}{ Valor de $\mathrm{p}$} & \multicolumn{3}{|c|}{ Valor de $p$} \\
\hline Estadística & Género & Estrato & Universidad & Género & Estrato & Universidad \\
\hline Wilks' Lambda & 0,2215 & 0,0005 & 0,0001 & 0,5359 & 0,2845 & 0,1219 \\
\hline Pillai's Trace & 0,2215 & 0,0005 & 0,0001 & 0,5359 & 0,2845 & 0,1219 \\
\hline Hotelling-Lawley T & 0,2215 & 0,0005 & 0,0001 & 0,5359 & 0,2845 & 0,1219 \\
\hline Roy's Greatest R & 0,2215 & 0,0005 & 0,0001 & 0,5359 & 0,2845 & 0,1219 \\
\hline
\end{tabular}

p >0,05 indica que no existe diferencia estadística, para las variables género, estrato y tipo de universidad.

Se utilizó análisis multidimensional de la varianza con contraste canónico ortogonal.

\section{TABLA 3}

Consumo expresado en porcentaje de derivados lácteos por género y estrato.

\begin{tabular}{|c|c|c|c|c|c|}
\hline & \multicolumn{2}{|c|}{ Género } & \multicolumn{3}{|c|}{ Nivel socioeconómico } \\
\hline & Masculino & Femenino & Bajo & Medio & Alto \\
\hline Yogurt & $87,2 \mathrm{a}$ & $82,1 \mathrm{a}$ & $91,3 \mathrm{a}$ & $80,4 b$ & $92,8 \mathrm{a}$ \\
\hline Kumis* & 60,9 a & $57,1 \mathrm{a}$ & $59,4 a$ & $57,8 \mathrm{a}$ & $62,5 \mathrm{a}$ \\
\hline Helados & $87,2 \mathrm{a}$ & $79,0 \mathrm{~b}$ & $76,8 \mathrm{~b}$ & $82,8 b$ & $89,2 \mathrm{a}$ \\
\hline Crema Leche & $98,0 \mathrm{a}$ & $58,1 \mathrm{~b}$ & $53,6 b$ & $58,3 \mathrm{~b}$ & $67,8 \mathrm{a}$ \\
\hline Lecherita* & $68,9 \mathrm{a}$ & $65,8 \mathrm{a}$ & $72,4 a$ & $66,8 \mathrm{~b}$ & $62,5 b$ \\
\hline Arequipe* & $75,0 \mathrm{a}$ & $67,8 \mathrm{~b}$ & $71,0 \mathrm{a}$ & $71,0 \mathrm{a}$ & $71,4 \mathrm{a}$ \\
\hline Postres & $34,1 \mathrm{a}$ & $34,1 \mathrm{a}$ & $28,9 b$ & $31,9 b$ & $50,0 \mathrm{a}$ \\
\hline Panelitas* & $57,9 a$ & $50,5 b$ & $57,9 a$ & $51,4 b$ & $58,9 a$ \\
\hline Jugos & $81,1 \mathrm{a}$ & $76,5 \mathrm{a}$ & $84,0 \mathrm{a}$ & $76,1 \mathrm{a}$ & $82,1 \mathrm{a}$ \\
\hline Manjares & $31,7 \mathrm{~b}$ & 39,6 a & $31,3 \mathrm{~b}$ & $34,0 \mathrm{~b}$ & $50,0 \mathrm{a}$ \\
\hline \multicolumn{6}{|c|}{$\begin{array}{l}\text { Letras distintas indican diferencia significativa por tipo de derivados lácteos entre géneros y entre niveles socioeconómicos }(p<0,05) \text {. } \\
\text { Se empleó el modelo lineal general (GLM). } \\
\text { * Kumis: derivado lácteo a partir kéfir de leche; Lecherita: leche condensada; } \\
\text { Arequipe: dulce de leche; Panelitas: dulce de leche de consistencia sólida elaborado con azúcar morena de caña y fécula de maíz. }\end{array}$} \\
\hline
\end{tabular}


por géneros (tabla 2).

El quesito, es el producto de mayor frecuencia (31\%) de consumo diario (tabla 4).

Se detectó una diferencia altamente significativa entre estratos y entre tipos de universidad pública y privada referente a la frecuencia de consumo de quesos $(p<0,001)$. La diferencia radicó entre el estrato alto y el bajo; no detectándose diferencia $(p=0,2215)$ entre géneros (tabla 2$)$.
Al indagar sobre el tipo de queso preferido, 87,2\% de los estudiantes lo prefieren fresco, 57,9\% semiduro, 57,6\% blando, $50,6 \%$ duro, $36,1 \%$ semigraso y $23,1 \%$ graso.

Al preguntar sobre el conocimiento de los nutrientes que aporta la leche, las mujeres manifestaron un mayor conocimiento frente al contenido de vitaminas y calcio, con diferencia significativa $(p<0,05)$ con respecto a los hombres (tabla 5$)$. Al efectuar el análisis multivariado de la varianza el cual evalúa

\section{TABLA 4}

Consumo de distintos tipos de queso por género y estrato.

\begin{tabular}{|c|c|c|c|c|c|c|c|c|}
\hline & \multicolumn{3}{|c|}{ Frecuencia } & \multicolumn{2}{|c|}{ Género } & \multicolumn{3}{|c|}{ Nivel socioeconómico } \\
\hline & Diario & Semanal & Rara vez & Masculino & Femenino & Bajo & Medio & Alto \\
\hline Queso & $31,0 b$ & $51,8 a$ & $17,1 c$ & $78,5 \mathrm{a}$ & $72,3 \mathrm{a}$ & $75,3 b$ & $72,9 b$ & $83,9 a$ \\
\hline Quesito* & $43,7 a$ & $41,4 a$ & $14,8 b$ & 83,5 a & $80,1 \mathrm{a}$ & $84,0 a$ & $83,4 a$ & $71,4 b$ \\
\hline Queso cuajada & $15,2 c$ & $39,5 b$ & $45,2 \mathrm{a}$ & $63,4 \mathrm{a}$ & $63,2 \mathrm{a}$ & $52,1 c$ & $63,8 b$ & $75,0 \mathrm{a}$ \\
\hline Queso pera & $5,2 c$ & $21,0 b$ & $73,6 a$ & $21,3 \mathrm{a}$ & 17,8 a & $17,3 b$ & $18,7 b$ & $25,0 a$ \\
\hline Queso amarillo & $4,4 c$ & $29,2 b$ & $66,2 \mathrm{a}$ & $22,5 \mathrm{a}$ & $24,5 \mathrm{a}$ & $23,1 b$ & $23,4 b$ & $40,5 a$ \\
\hline Queso doble crema & $5,2 \mathrm{c}$ & $25,0 b$ & $69,7 a$ & $27,4 \mathrm{a}$ & $25,5 \mathrm{a}$ & $20,2 b$ & $25,5 b$ & $37,5 a$ \\
\hline Queso fundido & $5,5 c$ & $37,3 b$ & $57,1 a$ & $37,8 \mathrm{a}$ & $34,1 \mathrm{a}$ & $21,7 b$ & $37,8 a b$ & $44,6 a$ \\
\hline Queso trenza & $2,3 c$ & $33,3 b$ & $64,2 \mathrm{a}$ & $11,5 \mathrm{a}$ & $7,6 \mathrm{a}$ & $7,2 b$ & $8,0 b$ & $17,8 a$ \\
\hline Queso mozzarella & $10,6 c$ & $52,2 \mathrm{a}$ & $37,0 b$ & $75,6 \mathrm{a}$ & $72,3 \mathrm{a}$ & $68,1 b$ & $72,2 b$ & $87,5 a$ \\
\hline Quesadillas & $5,2 c$ & $24,1 b$ & $70,5 a$ & $53,6 \mathrm{a}$ & 48,8 a & $46,3 b$ & $46,3 b$ & $58,9 a$ \\
\hline Queso ricota & $3,0 c$ & $21,2 b$ & $75,7 a$ & $9,1 \mathrm{a}$ & $7,6 \mathrm{a}$ & $4,3 b$ & $6,8 b$ & $19,6 a$ \\
\hline Queso gruyere & $6,4 c$ & $22,5 b$ & $70,9 a$ & $10,3 a$ & $5,6 \mathrm{~b}$ & $10,1 \mathrm{a}$ & $5,9 b$ & $12,5 a$ \\
\hline Queso parmesano & $6,3 c$ & $36,7 b$ & $56,8 a$ & $61,5 \mathrm{a}$ & 63,3 a & $55,8 b$ & $53,6 b$ & $73,2 a$ \\
\hline Queso gorgonzola & $9,5 c$ & $23,8 b$ & $66,6 a$ & $5,4 \mathrm{a}$ & $5,1 \mathrm{a}$ & $37,6 a$ & $4,2 c$ & $16,1 b$ \\
\hline Queso gouda & $4,1 c$ & $20,8 b$ & $75,0 a$ & $5,4 a$ & $5,6 \mathrm{a}$ & $4,3 b$ & $4,1 b$ & $21,4 a$ \\
\hline Queso roquefort & $11,5 c$ & $23,0 b$ & $65,3 a$ & $8,5 \mathrm{a}$ & $5,1 \mathrm{a}$ & $7,3 a$ & $6,8 a$ & $5,3 a$ \\
\hline Queso costeño & $8,1 c$ & $24,3 b$ & $67,5 a$ & $48,7 \mathrm{a}$ & $46,7 \mathrm{a}$ & $49,2 \mathrm{a}$ & $38,3 b$ & $50,0 a$ \\
\hline Queso para untar & $13,1 \mathrm{c}$ & $50,2 a$ & $36,6 b$ & $67,1 \mathrm{a}$ & $56,1 \mathrm{~b}$ & $63,7 a b$ & $58,3 b$ & $69,4 a$ \\
\hline
\end{tabular}

\section{TABLA 5}

Conocimientos sobre los nutrientes que aporta la leche.

\begin{tabular}{|c|c|c|c|c|c|}
\hline & \multicolumn{2}{|c|}{ Género } & \multicolumn{3}{|c|}{ Nivel socioeconómico } \\
\hline & Masculino & Femenino & Bajo & Medio & Alto \\
\hline Vitaminas & $84.5 b$ & $94.3 \mathrm{a}$ & $88.7 a$ & $90.6 \mathrm{a}$ & $92.4 \mathrm{a}$ \\
\hline Calcio & $83.6 b$ & $93.2 \mathrm{a}$ & $90.6 \mathrm{a}$ & $91.7 \mathrm{a}$ & $91.2 \mathrm{a}$ \\
\hline Zinc & $51.4 \mathrm{a}$ & $53.2 \mathrm{a}$ & $38.7 \mathrm{c}$ & $50.4 \mathrm{~b}$ & $62.4 \mathrm{a}$ \\
\hline Yodo & $15.8 \mathrm{a}$ & $18.1 \mathrm{a}$ & $11.3 \mathrm{~b}$ & $17.4 \mathrm{a}$ & $20.1 \mathrm{a}$ \\
\hline Magnesio & $34.6 \mathrm{a}$ & $36.2 \mathrm{a}$ & $26.4 \mathrm{~b}$ & $34.7 \mathrm{a}$ & $40.5 \mathrm{a}$ \\
\hline Cobre & $24.9 \mathrm{a}$ & $26.4 \mathrm{a}$ & $23.8 \mathrm{a}$ & $24.7 \mathrm{a}$ & $25.4 \mathrm{a}$ \\
\hline Grasas & $82.4 \mathrm{a}$ & $85.7 \mathrm{a}$ & $83.9 \mathrm{a}$ & $84.5 \mathrm{a}$ & $87.6 \mathrm{a}$ \\
\hline Proteínas & $87.3 \mathrm{a}$ & $87.7 \mathrm{a}$ & $85.4 \mathrm{a}$ & $83.1 \mathrm{a}$ & $88.1 \mathrm{a}$ \\
\hline
\end{tabular}

Letras distintas indican diferencia significativa por tipo de nutriente entre géneros y entre niveles socioeconómicos $(p<0,05)$.

Se empleó el modelo lineal general (GLM). 
todas las variables de manera simultánea, no se detectó una diferencia estadística ( $p>0.05)$ por género, estrato y tipo de universidad referente al conocimiento que poseen sobre los distintos componentes que posee la leche (tabla 2).

Al indagar sobre su consumo en la infancia, 85,2\% consumían leche con regularidad en su infancia, 13,3\% rara vez y $2,5 \%$ nunca. Al indagar las razones para no consumir productos lácteos, 28,3\% de los estudiantes respondieron que no consumían lácteos por su alto costo, 20,9\% por su sabor y $15,4 \%$ por factores de salud relacionados con intolerancia a lactosa (tabla 6).

\section{DISCUSIÓN}

Se encontró en la población universitaria una alta frecuencia de consumo de leche entera $(60,2)$ y de quesito $(43,8 \%)$ y un conocimiento bueno del aporte de calcio de los productos lácteos, entre hombres y mujeres, $83.6 \%$ y $93.2 \%$ respectivamente. Aunque el estudio no indagó sobre la cantidad de consumo y los conocimientos sobre el requerimiento diario de consumo en población universitaria, un estudio en población escolar de la ciudad de Medellín (14) encontró un consumo de 135,5 L/persona/año, bajo con respecto a la recomendación de la OMS de 180 L/persona/año, a su vez se encontró un consumo alto de gaseosas y refrescos. Esto sugiere la necesidad de implementar campañas educativas orientadas a aumentar su consumo, teniendo en cuenta el alto conocimiento encontrado en el presente estudio sobre los beneficios de los lácteos para la salud, que coincide con los hallazgos de otros autores (15), aspecto relacionado con las políticas de desarrollo de la cadena láctea colombiana y la necesidad de proveer una mayor educación a los consumidores enfocada a incrementar su consumo (16).

En cuanto a la preferencia en consumo de bebidas, los hallazgos indican que los universitarios tienen mayor preferencia por la gaseosa (9,5\%) que por la leche $(6,1 \%)$, siendo más costosa la gaseosa, por lo cual se deduce que el precio no es el factor decisivo; razón por la cual se plantea la necesidad de realizar campañas de educación en nutrición, orientadas a reducir el consumo de bebidas gaseosas en beneficio del consumo de leche y jugos de frutas, teniendo en cuenta que la publicidad en televisión de las gaseosas es un factor que aumenta la propensión a su consumo (17). Estos programas deben enfocarse en mensajes de alimentación saludable (18-20), orientados a la cantidad diaria que se debe consumir (21).

Respecto a las razones para no consumir lácteos, los hallazgos indican que el sabor es un factor importante, lo cual coincide con otros estudios (22), razón por la cual es importan- te tener en cuenta el desarrollo de nuevos sabores como factor motivacional para incrementar el consumo. Adicionalmente, se debe considerar en la promoción del consumo, los productos preferidos como acompañantes de la leche identificados en el estudio, entre ellos el café, los jugos y los dulces.

Los hallazgos del estudio indican que el hogar es el sitio en el cual se recalca la importancia del consumo de lácteos, lo cual concuerda con otros estudios que han encontrado que los padres ejercen una influencia positiva en la ingesta de lácteos por parte de niños y adolescentes, al permitir su disponibilidad y tener creencias favorables sobre sus beneficios para la salud $(23,24)$, por lo cual deben incluirse en las campañas de promoción (25), orientadas a la necesidad de cambiar los hábitos de consumo, para alcanzar los requerimientos nutricionales.

\section{CONCLUSIONES}

Teniendo en cuenta las recomendaciones de consumo diario de lácteos de la OMS que equivale a 3 porciones diarias, la frecuencia diaria de consumo de los universitarios que alcanza sólo al 60,2\% es baja y requiere estrategias de promoción enfocadas en la educación nutricional que contribuyan a lograr cambios de comportamiento e influenciar la elección de dietas saludables, especialmente en las mujeres que consumen menos productos lácteos que los hombres.

Estas estrategias de promoción deben apoyar el mensaje ya conocido por un alto porcentaje de los estudiantes que conoce la importancia del aporte de calcio de los productos lácteos, a partir de la ventaja de ser un producto de menor valor comercial que las bebidas sustitutas, brindando alternativas de sabor y de productos bajos en grasa o deslactosados que superen los problemas de salud argumentados para no consumir lácteos.

\section{RESUMEN}

Se evaluó el consumo de productos lácteos en una población de 420 estudiantes universitarios de 4 instituciones de educación superior de la ciudad de Medellín, Colombia, para determinar las preferencias y conocimientos frente al consumo por medio de una entrevista personal. Se efectuó el análisis de los datos aplicando la técnica multivariada MANOVA con contraste canónico ortogonal empleando el paquete estadístico SAS versión 9.0. Se encontró que un $80,7 \%$ de los universitarios consumen leche, con una diferencia altamente significativa en la frecuencia de consumo entre niveles socioeconómicos, siendo el nivel alto el de mayor consumo. Se encontró una diferencia significativa en el consumo de lácteos entre sexos, con un mayor consumo por parte de los hombres. La leche ocupa el quinto lugar como bebida preferida $(6,1 \%)$,

\section{TABLA 6}

Razones para no consumir lácteos.

\begin{tabular}{|c|c|c|c|c|c|}
\hline \multirow[b]{2}{*}{ Razón } & \multicolumn{2}{|c|}{ Género } & \multicolumn{3}{|c|}{ Estratos } \\
\hline & Masculino & Femenino & Bajo & Medio & Alto \\
\hline Salud & $16.7 \mathrm{a}$ & $14.3 \mathrm{a}$ & $15.4 \mathrm{a}$ & $13.4 \mathrm{a}$ & $11.2 \mathrm{a}$ \\
\hline Precio & $30.4 \mathrm{a}$ & $26.2 \mathrm{a}$ & $35.6 \mathrm{a}$ & $18.7 \mathrm{~b}$ & $10.4 \mathrm{c}$ \\
\hline Sabor & $20.2 \mathrm{a}$ & $19.3 \mathrm{a}$ & $23.5 \mathrm{a}$ & $18.7 \mathrm{a}$ & $21.3 \mathrm{a}$ \\
\hline Otros & $35.4 \mathrm{a}$ & $33.9 \mathrm{a}$ & $35.8 \mathrm{a}$ & $32.7 \mathrm{a}$ & $33.4 \mathrm{a}$ \\
\hline
\end{tabular}

Letras distintas indican diferencia significativa por razones para no consumir lácteos entre géneros y entre niveles socioeconómicos ( $\mathrm{p}<0,05)$.

Se empleó el modelo lineal general (GLM). 
por detrás de jugos, agua, gaseosa y aromáticas. Las razones principales para no consumir lácteos son el costo $(28,3 \%)$ y el sabor $(20,9 \%)$.

Palabras clave: consumo de lácteos, estudiantes universitarios, hábitos alimentarios.

\section{BIBLIOGRAFIA}

1. Berterreche J. Aspectos nutracéuticos y funcionales en productos lácteos. Consultado el 26/04/2011 Disponible en: http://www.fepale.org/sitio_viejo/ lechesalud/documentos/Javier\%20Berterreche\%20 Alim\%20Funcionales.pdf

2. Huncharek M. Muscat J. Kupelnick B. Impact of dairy products and dietary calcium on bone-mineral content in children: Results of a meta-analysis. Bone 2008; 43:312-21.

3. Shin H. Yoon YS. Lee Y. Kim C. Oh SW. Dairy Product Intake Is Inversely Associated with Metabolic Syndrome in Korean Adults: Anseong and Ansan Cohort of the Korean Genome and Epidemiology Study Endocrinology. J Korean Med Sci. 2013; 28:1482-8.

4. Weaver C. Role of dairy beverages in the diet. Physiol Behav. 2010; 100:63-6.

5. Abreu S. Moreira P. Moreira C. Mota J. Moreira-Silva I. Santos P. Santos R. Intake of milk, but not total dairy, yogurt, or cheese, is negatively associated with the clustering of cardiometabolic risk factors in adolescents. Nutr Res. 2014; 34:48-57.

6. Yuan W. Kakinami L. Gray-Donald K. Czernichow S. Lambert M. Paradis G. Influence of Dairy Product Consumption on Children's Blood Pressure: Results from the QUALITY Cohort. J Academy Nutr Dietetics 2013; 113:936-41.

7. Digioia K. Lim C. Janicke D. Mathews A. Dairy Intake Is Associated with Adiposity in Overweight and Obese Children Living in Rural Areas. J Acad Nutr Diet 2012; 112:A16.

8. Tanaka K. Miyake Y. Sasaki S. Intake of dairy products and the prevalence of dental caries in young children. J Dentistry 2010; 38:579-83.

9. Colombia. Departamento Nacional de Planeación. Política nacional para mejorar la competitividad del sector lácteo colombiano. Documento Conpes 3675. Consultado el 13/04/2011 Disponible en: http://www.minagricultura .gov.co/archivos/conpes_3675_sector_lacteo.pdf

10. OMS. Necesidades de calcio: informe de un grupo mixto FAO/OMS de expertos. Serie de Informes técnicos $N^{\circ} 230$. Consultado el 15/04/2011 Disponible en: http://whqlibdoc.who.int/trs/WHO_TRS_230_spa.pdf

11. Colombia. Instituto Colombiano de Bienestar Familiar, ICBF. Encuesta nacional de la situación nutricional en Colombia - ENSIN, 2005. Consultado el 20/04/2013 Disponible en: http://www.icbf.gov.co/portal/page/portal/Portal/CBF /NormatividadC/ENSIN1/ENSIN2005/LIBRO_2005.pdf

12. Poddar K. Hosig K. Anderson-Bill E. Nickols-Richardson S. Duncan S. Dairy Intake and Related Self-Regulation Improved in College Students Using Online Nutrition Education. J Academy Nutr Diet 2012; 112:1976-86.
13. Colombia. Instituto Colombiano de Bienestar Familiar, ICBF. Encuesta nacional de la situación nutricional en Colombia - ENSIN, 2010. Consultado el 20/04/2013 Disponible en: http://www.icbf.gov.co/portal/page/portal/Portal/CBF /NormatividadC/ENSIN1/ENSIN2010/LibroENSIN2010.pdf

14. Rodríguez H. Restrepo L. Martínez L. Conocimientos, gustos y prácticas sobre el consumo de lácteos en una población escolar de la ciudad de Medellín-Colombia. Perspectivas Nutr Hum. 2014; 16:83-96.

15. Utter J. Scragg R. Schaaf D. Associations between television viewing and consumption of commonly advertised foods among New Zealand children and young adolescents. Public Health Nutr. 2006; 9:606-12.

16. Colombia. Ministerio de Agricultura y Desarrollo Rural. Agenda prospectiva de investigación y desarrollo tecnológico de la cadena láctea colombiana. Consultado el 13/04/2011 Disponible en: http://www.agronet.gov.co/www/docs _agronet/200831311504_L\%E1cteos.pdf

17. Harnack L. Stang J. Story M. Soft Drink Consumption Among US Children and Adolescents: Nutritional Consequences. J Am Diet Assoc. 1999; 99:436-41.

18. Jacoby E. Keller I. La promoción del consumo de frutas y verduras en américa latina: buena oportunidad de acción intersectorial por una alimentación saludable. Rev Chil Nutr. 2006; 33:226-31.

19. Costa L. Vasconcelos F. Corso A. Fatores associados ao consumo adequado de frutas e hortaliças em escolares de Santa Catarina, Brasil. Cadernos Saúde Pública 2012; 28:1133-42.

20. Reger B. Wootan M. Booth-Butterfield S. Smith H. 1\% or less: a community-based nutrition campaign. Department of Community Medicine, West Virginia University, Morgantown, USA. Public Health Rep. 1998; 113:410-19.

21. Griffin M. Campaña panamericana de consumo de lácteos: sí a la leche "por la salud de nuestros niños". Consultado el 26/04/2011 Disponible en: http://www.sialaleche.org/ pdf/Michael_Griffin.pdf

22. De Pelsmaeker S. Schouteten J. Gellynck X. The consumption of flavored milk among a children population. The influence of beliefs and the association of brands with emotions. Appetite 2013; 71:279-86.

23. Olson B. Chung K. Reckase M. Schoemer S. Parental Influences on Dairy Intake in Children, and Their Role in Child Calcium-fortified Food Use. J Nutr Education Behav 2009; 41:53-7.

24. Kranz S. Lin P. Wagstaff D. Children's Dairy Intake in the United States: Too Little, Too Fat? J Pediatr. 2007; 151:642-6.

25. Fulgoni V. Nicholls J. Reed A. Buckley R. Kafer K. Huth P. Dirienzo D. Miller G. Dairy Consumption and Related Nutrient Intake in African-American Adults and Children in the United States: Continuing Survey of Food Intakes by Individuals 1994-1996, 1998, and the National Health and Nutrition Examination Survey 1999-2000. J Am Diet Assoc. 2007; 107:256-64. 